\title{
EXPRESIÓN DE MOLÉCULAS B7 Y TLR:9 EN CÉLULAS EPITELIALES CORNEALES INFECTADAS CON ADENOVIRUS: IMPLICACIONES CLÍNICO-PATOLÓGICAS EN LA QUERATOCONJUNTIVITIS VIRAL
}

\author{
EXPRESSION OF B7 MOLECULES AND TLR-9 ON CORNEAL \\ EPITHELIAL CELLS INFECTED WITH ADENOVIRUS: CLINICO- \\ PATHOLOGICAL IMPLICATIONS IN VIRAL \\ KERATOCONJUNCTIVITIS
}

\author{
JIMÉNEZ-MARTÍNEZ MC ${ }^{1}$, MEJÍA H ${ }^{2}$, LINARES M², SANTACRUZ C ${ }^{3}$, SÁNCHEZ-NAVARRO A ${ }^{3}$, \\ SUÁREZ R ${ }^{3}$, GARFIAS Y ${ }^{1}$
}

\section{RESUMEN}

Objetivo: Las moléculas B7 son una familia de proteínas que coestimulan al linfocito $\mathrm{T}$ durante la activación inmunitaria, normalmente las células epiteliales corneales (CEC) no expresan estas moléculas en superficie. Los receptores tipo Toll juegan un papel importante en la respuesta inmune innata hacia patógenos invasores y recientemente se demostró su expresión en córneas de ratón. El objetivo del presente estudio fue determinar si la infección viral induce moléculas B7 y TLR9 en CEC humanas.

Métodos: Las CEC fueron obtenidas de corneas humanas tratadas con dispasa II y crecidas en presencia de medio hormonal epitelial suplementado hasta su confluencia. Posteriormente las células

\begin{abstract}
Purpose: B7 molecules are a family of proteins that co-stimulate $T$ cells during immune activation. Normally the corneal epithelial cells (CEC) do not express these molecules on their cell surface. Tolllike receptors play an important role in the innate immune response to invading pathogens and recently have been demonstrated to be expressed on mice cornea. The objective of this study was to determine whether adenoviral infection induces B7 molecules and TLR9 on human CEC.

Methods: CEC were isolated from human corneas treated with dispase-II, and grown in the presence of supplemented hormonal epithelial medium until confluence. Then CEC were then infected with adenovirus 5 (Ad5) and cultured for different times.
\end{abstract}

Recibido: 20/10/05. Aceptado: 4/7/06.

Instituto de Oftalmología Fundación Conde de Valenciana. Unidad de Investigación y Departamento de Córnea. México, D.F. México.

1 Doctor en Medicina. Doctor en Inmunología.

2 Licenciada en Medicina.

3 Doctor en Medicina.

Este trabajo fue apoyado parcialmente por «Fundación de Asistencia Privada Conde de Valenciana, IAP» y CONACYT Proyecto Salud-2004C01-96.

Correspondencia:

María C. Jiménez-Martínez

Instituto de Oftalmología Fundación Conde de Valenciana

Unidad de Investigación

Chimalpopoca Núm. 14

Col. Obrera, C.P. 06800

Del. Cuauhtémoc

México, D.F.

E-mail: jmaricarmen@yahoo.com 
fueron infectadas con adenovirus 5 (Ad5) y cultivadas a diferentes tiempos. Las CEC fueron recuperadas y marcadas contra CD80, CD86, TLR-9 y citoqueratina. Todas las células fueron analizadas por citometría de flujo.

Resultados: La infección de CEC con Ad5 indujo la expresión de moléculas B7 y TLR-9 desde las 24 h, alcanzando su máximo nivel a las $72 \mathrm{~h}$. La expresión de moléculas B7 a las $72 \mathrm{~h}$ fue como sigue, expresión de CD80 en CEC infectadas 62\% error estándar (ES) 2.6 versus 3 ES $1.2(\mathrm{p}<0,001)$ en CEC no infectadas; expresión de CD86 en CEC infectadas $95 \%$ ES 2.1 versus 5\% ES 1.2 (p < 0,001) en CEC no infectadas. La expresión de TLR9 a las $72 \mathrm{~h}$ fue de $80 \%$ ES 1.2 en CEC infectadas versus 5\% ES 1 en CEC no infectadas ( $p<0,001$ ). Conclusiones: La infección por Ad5 induce la expresión de moléculas B7 y TLR-9 en CEC.

Palabras clave: B7, TLR-9, queratoconjuntivitis adenoviral, células epiteliales corneales.
The CEC were then recovered and stained against human CD80, CD86, TLR-9 and cytokeratin. All cells were analyzed by flow cytometry.

Results: Ad5 infection of CEC induced the expression of B7 molecules and TLR-9 after 24 hours in culture, rising to maximum levels at 72 hours. B7 expression at 72 hours was as follows: CD80 expression on infected CEC was 62\% (standard error [SE] 2.6) versus 3\% (SE 1.2) on non-infected CEC ( $\mathrm{p}<0.001)$; CD86 expression on infected CEC was 95\% (SE 2.1) versus 5\% (SE 1.2) on non-infected CEC $(\mathrm{p}<0.001)$. TLR-9 expression at 72 hours was $80 \%$ (SE 1.2) on infected CEC versus $5 \%$ (SE 1) on non-infected CEC $(p<0.001)$.

Conclusions: Ad5 infection induced the expression of B7 molecules and TLR-9 on CEC (Arch Soc Esp Oftalmol 2006; 81: 391-400).

Key words: B7, TLR-9, adenoviral keratoconjunctivitis, corneal, epithelial cells.

\section{INTRODUCCIÓN}

El ojo es un lugar inmunoprivilegiado porque tiene una compleja red de mecanismos que mantienen la tolerancia inmunológica ante antígenos (Ag) propios (1). Entre los componentes que regulan la respuesta inmunitaria se incluyen la ignorancia inmunológica, en la que las células del epitelio corneal (CEC) no permiten el desarrollo de vasos sanguíneos y linfáticos en su superficie, impidiendo el acceso de linfocitos activados (2), si esto ocurriera, las células del endotelio corneal pueden inducir muerte celular programada a los linfocitos autoreactivos (3). Otro mecanismo de tolerancia inmunológica es la carencia de moléculas del complejo principal de histocompatibilidad (MHC) y de moléculas que favorecen la respuesta inmunitaria específica, a través de la coestimulación al linfocito T (4).

La función de las moléculas coestimulatorias es aumentar la señalización durante la activación linfocitaria (5); normalmente las células corneales no expresan moléculas coestimulatorias, sin embargo se ha demostrado que en el estroma corneal, las células dendríticas y las células de Langerhans (6), pueden expresar moléculas coestimulatorias de la familia B7, como CD80 o CD86, ante un estímulo antigénico y funcionar como células presentadoras de antígeno durante un rechazo corneal, sugiriéndo- se que su maduración funcional está implicada con el desarrollo de la respuesta inmunitaria (7). En este mismo modelo, se ha demostrado que las células dendríticas corneales producen factor de crecimiento transformante beta (TGF- $\beta$ ) (8), que regula negativamente la expresión de moléculas coestimulatorias y la activación de linfocitos $\mathrm{T}$, induciendo un microambiente inmunosupresor (9-10).

Una infección puede modificar el microambiente ocular y causar daño a la superficie ocular; entre las infecciones más frecuentes se encuentran las causadas por adenovirus (Ad) (11). Se ha demostrado que durante la infección por Ad las células CEC infectadas tienen la capacidad de producir interleucina-1 (IL-1) (12). Algunos autores han señalado que la IL1 es capaz de inducir moléculas B7 en células epiteliales de otros sitios anatómicos como la piel (13) o el epitelio respiratorio (14). Además, en modelos animales de infección viral, existe un incremento de TGF- $\beta$ en el microambiente, que funcionaría como un agente antiinflamatorio con propiedades antivirales (15). Por otra parte, se ha observado que durante la respuesta inmunitaria innata frente a los virus, algunas células epiteliales expresan moléculas que reconocen patrones moleculares conservados, a este tipo de estructuras se les conoce como receptores tipo Toll (TLR) y se ha demostrado que el TLR-9 puede interactuar con $\mathrm{CpG}$ no metilado presente en 
ADN bacteriano o viral (16). El objetivo principal de este estudio fue determinar si la infección con adenovirus era capaz de inducir en CEC la expresión de moléculas que colaboran con la respuesta inmunitaria específica (moléculas B7) o con la respuesta inmunitaria innata (TLR-9).

\section{SUJETOS, MATERIAL Y MÉTODOS}

\section{Obtención de Células Epiteliales Corneales}

Las CEC fueron obtenidas a partir de botones corneales humanos libres de infección viral provenientes de individuos sometidos a transplante corneal secundario a queratocono. Los datos presentados provienen de CEC expandidas a partir de 3 botones corneales de individuos no relacionados y de cada expansión celular se hicieron ensayos por triplicado. Las CEC fueron separadas del estroma con dispasa II (Roche, Germany) durante $15 \mathrm{~min}$ a $37^{\circ} \mathrm{C}$, las CEC fueron separadas de las estromales mediante una malla de Nylon. Una vez obtenidas fueron lavadas con medio DMEM (Dulbecco Modified Eagle Medium) (Gibco, Grand Island, NY, USA) y se valoró su viabilidad con la exclusión de azul tripano. Las células se expandieron en presencia de medio epidermal hormonal suplementado (SHEM) en cajas de 24 pozos (Costar, NY, USA). El SHEM consiste en DMEM/F12 v/v (Gibco, Grand Isalnd, NY, USA), factor de crecimiento epidermal (Sigma, St. Louis Missouri, USA), insulina, tranferrina, selenio (Roche, Mannheim, Alemania), hidrocortisona, gentamicina, estreptomicina, penicilina, anfotericina B (Sigma, St. Louis Missouri, USA) y $10 \%$ de suero fetal bovino (Gibco, Grand Island, NY, USA) (17). Cuando las células alcanzaron un $80 \%$ de confluencia, aproximadamente de 15-20 días después, fueron despegadas de los pozos con tripsina al 0,25\% (Gibco, Grand Island, NY, USA) y se pasaron a una caja de $25 \mathrm{~mm}^{2}$ (Costar, NY, USA). Ahí, se cultivaron de 17-20 días adicionales, en las mismas condiciones de cultivo, hasta que alcanzaron una confluencia del $90 \%$, utilizándose para los ensayos posteriores.

\section{Extracción de ADN y Reacción en cadena de la Polimerasa (PCR)}

Para la extracción de ADN en tejidos, se siguió la metodología propuesta por el fabricante con el mini kit QIAamp (QIAGEN Sciences, Maryland, USA). Para asegurar que los botones corneales estuvieran libres de infección viral previa se realizó PCR para identificación de adenovirus o herpesvirus. Los cebadores para adenovirus fueron ADRJC1, 5'GACATGACTTTCGAGGTCGATCCCATGGA3'; secuencia antisentido ADRJC2, 5'-CCGGCTGAGAAGGGTGTGCGCAGGTA-3', amplificando un producto de $140 \mathrm{pb}$; para herpesvirus fueron ALE1 5'-TTATTGCCGTCATAGCGCGG-3'; secuencia antisentido ALE2 5'-GGCGACCTGTATAACGTGTT-3', amplificando un producto de 278 pb. La PCR se realizó con el kit Omniscrpt Reverse Transcription (QIAGEN Sciences, Maryland, USA), la amplificación con un termociclador GeneAmp PCR System 2400 (Perkin Elmer Co. Norwalk, Connecticut, USA). Los botones corneales positivos por PCR para adenovirus y/o herpesvirus se eliminaron del estudio.

\section{Identificación de Ad5}

El Ad5 fue identificado mediante enzimas de restricción Eco-RI, Hind-III, Bgl-II y Sma-I de acuerdo a las instrucciones del fabricante (Sigma Chemical Co, Saint Louis Mo, USA). Los productos de restricción fueron observados en una electroforesis de agarosa al $1 \%$, corridos durante 9 h a 40 volts. Las bandas de ADN fueron teñidas con bromuro de etidio al $0,1 \%$, utilizando marcadores de peso molecular $\lambda$ у $\varphi$ (Promega, Madison, WI, USA). El patrón de restricción fue comparado con perfiles previamente publicados para adenovirus (18).

\section{Titulación de partículas virales}

Células Vero (American Type Culture Collection, CRL-1586) fueron infectadas con diluciones seriadas de Ad5 hasta observar un efecto citopático. Las partículas infectantes se determinaron por el método de Reed-Muench (19), siendo el título de virus inoculados de 6,08 X108/100 ml.

\section{Cultivos celulares}

Se realizaron cultivos celulares en presencia y ausencia de Ad5. En la infección de las CEC se utilizó el título viral obtenido en células vero. Las 
CEC fueron recuperadas en intervalos de $24 \mathrm{~h}$ hasta el tercer día. El sobrenadante (SN) de cultivo fue congelado a $-70^{\circ} \mathrm{C}$ hasta su utilización.

\section{Inmunofluorescencia}

Las CEC recuperadas de cultivo fueron procesadas para citometría de flujo, utilizando anticuerpos monoclonales conjugados a un fluorocromo y dirigidos contra CD80, CD86 (BD Biosciences Pharmingen, CA, USA), TLR-9 (e-Biosciences, CA, USA) y citoqueratina, clona MNF116 (DAKO, Carpintería, CA, USA).

\section{Determinación de citocinas}

El SN de los cultivos fue utilizado para determinar TGF- $\beta$ mediante un sándwich ELISA cuantitativo, según la metodología propuesta por el fabricante (R\&D Systems, MN, USA). Los resultados fueron leídos en un lector de ELISA (TermoLabsystems, Helsinki, Finland) a 450nm de longitud de onda y corrección de $540 \mathrm{~nm}$.

\section{Análisis Estadístico}

Se utilizó U-Mann Withney para comparación entre dos grupos, debido a que al realizar el análisis de normalidad con la prueba de Kolmogorov-Smimov se determinó que nuestros datos no tenían una distribución normal. Se consideró una $\mathrm{p}<0.05$ como estadísticamente significativa. El análisis estadístico se realizó con el programa SigmaStat (Systat Software, Inc. Point Richmond, CA, USA. Versión 10) y las gráficas con SigmaPlot (Systat Software, Inc. Point Richmond, CA, USA. Versión 10).

\section{RESULTADOS}

\section{Infección de Células Epiteliales Corneales por Adenovirus 5}

El primer objetivo de este estudio fue evaluar la infección de Ad5 en CEC. La infección viral fue demostrada observando el efecto citopático característico (fig. 1a), por inmunofluorescencia directa (fig. 1b), por PCR (fig. 1c); la figura 1d muestra el patrón característico de corte por enzimas de restricción de Ad5.

\section{Cambios morfológicos en las células cultivadas}

Las CEC en cultivo fueron perdiendo sus características morfológicas epiteliales clásicas adquiriendo una forma fibroblástica, independientemente de si estaban infectadas o no por Ad5, por lo que fue necesario determinar la expresión de citoqueratina, característica fenotípica clásica de las células epiteliales (20), utilizando anticuerpos anti-citoqueratina conjugada a un fluorocromo. En la figura 2 (a) se observan las características de tamaño (FSC$\mathrm{H})$ y de granularidad (SSC-H) que tienen las CEC en una citometría de flujo. En la figura 2 (b) se observa que aproximadamente el $70 \%$ de las CEC mantiene sus características fenotípicas epiteliales, siendo Citoqueratina (CK) positivas (+); mientras que aproximadamente el $30 \%$ de las células que por granularidad y tamaño corresponden con CEC pierden la expresión de este marcador [30\% error estándar (ES) 5,2]. Los porcentajes de CEC CK+ fueron similares en las células infectadas $(70 \%$ ES 6,4) y no infectadas $(72 \%$ ES 4,3); no se encontraron diferencias estadísticamente significativas entre ellas.

\section{Expresión de moléculas B7 en CEC infectadas con Ad5}

La cinética de expresión de moléculas B7 (CD80 y CD86) fue evaluada en las CEC CK+, infectadas y no infectadas con Ad5. Los datos obtenidos muestran que el porcentaje de CEC CK+ CD80+ fue de $54 \%$ a las $24 \mathrm{~h}, 52,3 \%$ a las $48 \mathrm{~h}, 62 \%$ a las $72 \mathrm{~h}$ (fig. 3 ); en contraste el porcentaje para las CEC CK+ CD80+ no infectadas fue $<5 \%$ en los tres días de cultivo (fig. 3, tabla I). La cinética de expresión de CD86 en CEC CK+ siguió un comportamiento similar, siendo de $62,3 \%$ a las $24 \mathrm{~h}, 86 \%$ a las $48 \mathrm{~h}$ y $95 \%$ a las 72 h (fig. 3, tabla I).

\section{Expresión de TLR-9 en CEC infectadas con Ad5}

La cinética de expresión de TLR-9 fue evaluada en las CEC CK+, infectadas y no infectadas con Ad5. Los datos obtenidos muestran que el porcen- 
(a)

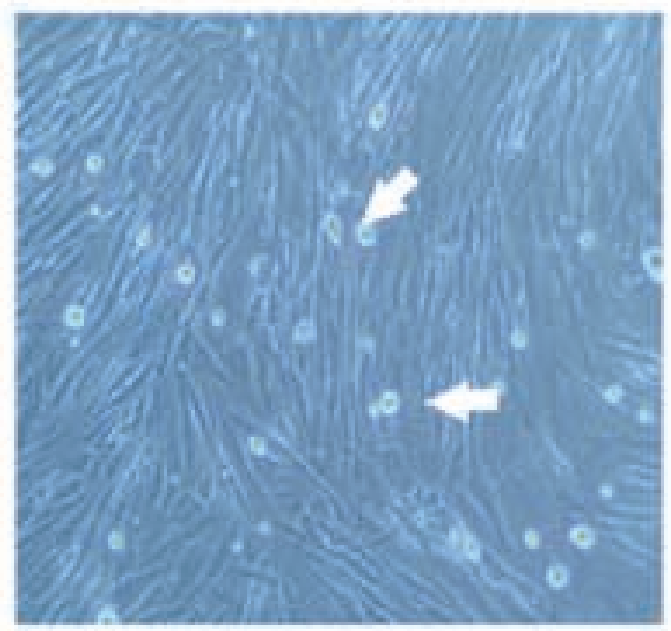

(c)

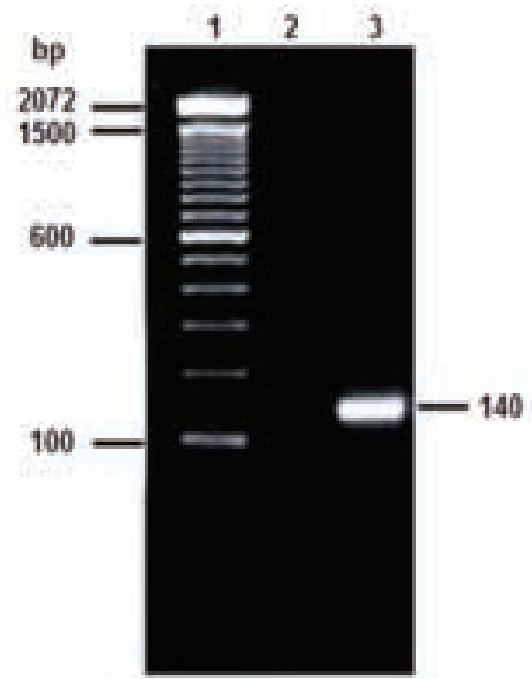

(b)

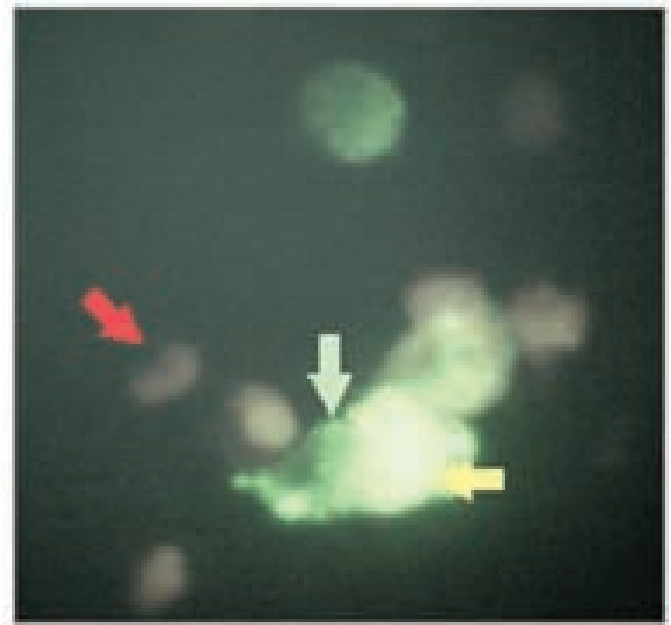

(d)

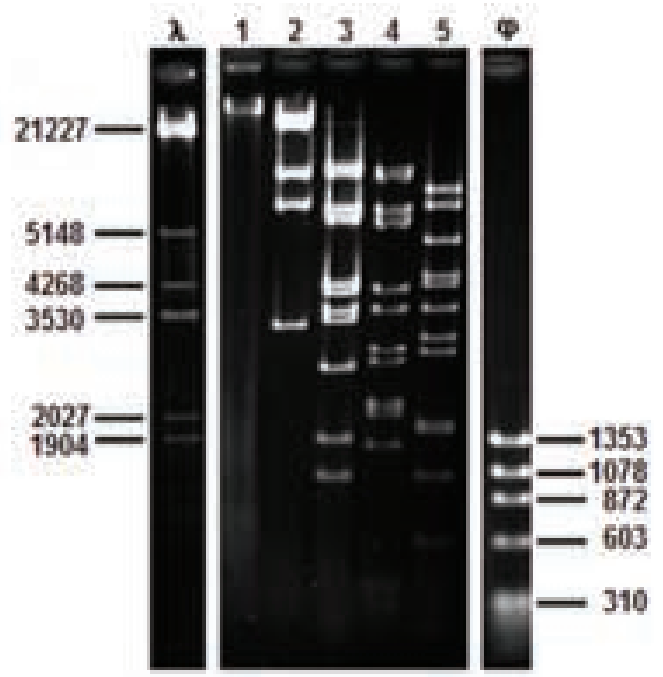

Fig. 1: Evaluación de la infección de Ad5 en CEC. En (a) se observa el efecto citopático del virus (flechas blancas) en las células en cultivo, magnificación 10x; En (b) se observa la inmunofluorescencia positiva a la tinción con anticuerpos anti-adenovirus conjugados a FITC (Adenovirus DFA kit, CHEMICON international, USA) en células infectadas (flecha verde) y la tinción nuclear con yoduro de propidio, en células no infectadas (flecha roja), la colocación de ambas tinciones da lugar a un color amarillo (flecha amarilla), magnificación $40 x$. En (c) se observa PCR para adenovirus; el carril 1 corresponde a los pesos moleculares, el carril 2 a las células no infectadas, el carril 3 a las células infectadas, en este carril se encuentra un producto de 140 bp que corresponde al amplificado de los cebadores ADRCJ1, ADRCJ2 utilizados para la identificación viral, tinción con bromuro de etidio. En (d) se observa el patrón de restricción que caracteriza a Ad5; el carril 1 corresponde al ADN sin cortar, el carril 2 corresponde al ADN cortado con Eco-RI, el carril 2 corresponde al ADN cortado con Hind-III, el carril 4 corresponde al ADN cortado con Bgl-II, el carril 5 corresponde al ADN cortado con Sma-I; $\lambda$ y $\varphi$ corresponden a marcadores moleculares. 
(a)

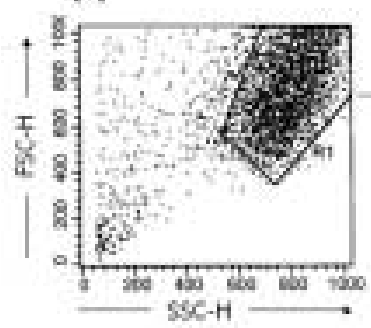

(b)

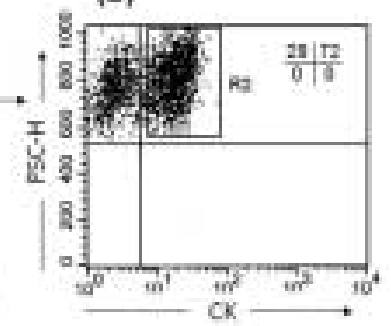

Fig. 2: Características citométricas de las CEC. En (a) se observan las características de tamaño (FSC) y granularidad (SSC) de las células cultivadas. En (b) se muestra una gráfica de puntos que proviene de la región 1 «Rl» mostrada en (a). Rl incluye exclusivamente a las células que por tamaño y granularidad corresponden con CEC. R2 señala las células CK+. FSC en el eje de las «y», tinción con anticuerpo anti-citoqueratina conjugada a un fluorocromo en el eje de las «x». taje de CEC CK+ TLR-9+ fue de $62 \%$ a las $24 \mathrm{~h}$, $63,3 \%$ a las 48 h y $80 \%$ a las 72 h (fig. 4); en contraste, el porcentaje de las CEC CK+ TLR-9+ no infectadas fue alrededor del $5 \%$ en los tres días de cultivo (fig. 4 , tabla II).

\section{TGF- $\beta$ en el sobrenadante de las CEC cultivadas}

La producción TGF- $\beta$ fue evaluada por ELISA durante los diferentes días de cultivo. Los resultados mostraron un incremento en la producción de TGF-b detectado en el sobrenadante de cultivo de las CEC infectadas por Ad5, en comparación con las CEC no infectadas (fig. 5), sin embargo las diferencias cuantitativas encontradas no fueron estadísticamente significativas.

Tabla I. Cinética de expresión de CD80 y CD86 en CEC CK+ durante los diferentes días de cultivo

\begin{tabular}{|c|c|c|c|c|c|c|}
\hline & \multicolumn{3}{|c|}{ CD80 } & \multicolumn{3}{|c|}{ CD86 } \\
\hline & $24 \mathrm{~h}$ & $48 \mathrm{~h}$ & $72 \mathrm{~h}$ & $24 \mathrm{~h}$ & $48 \mathrm{~h}$ & $72 \mathrm{~h}$ \\
\hline CEC Infectadas & $54 \pm 13,8$ & $52,3 \pm 12,2 *$ & $62 \pm 2,6 * *$ & $62,3 \pm 10,1$ & $86 \pm 3,6^{* *}$ & $95 \pm 2,1 * *$ \\
\hline CEC No infectadas & $2 \pm 1$ & $4 \pm 1,5^{*}$ & $3 \pm 1,2 * *$ & $3,3 \pm 1,1$ & $4 \pm 1,6 * *$ & $5 \pm 1,2 * *$ \\
\hline
\end{tabular}

Resultados expresados en porcentaje \pm error estándar. *: $\mathrm{p}=0,017 ; * *$ : $\mathrm{p}<0,001$.

\section{CEC CK+}

(a)

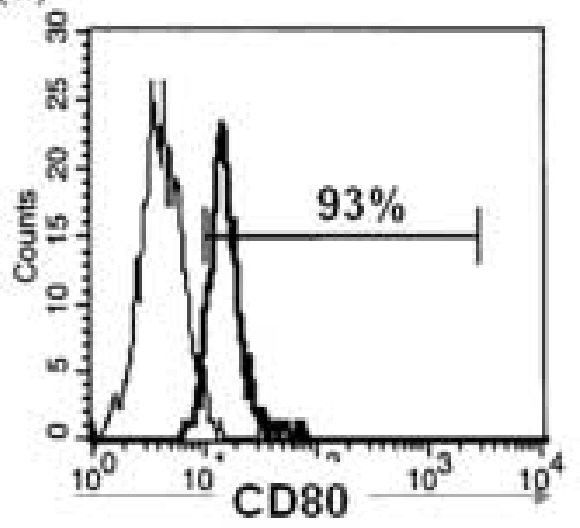

(b)

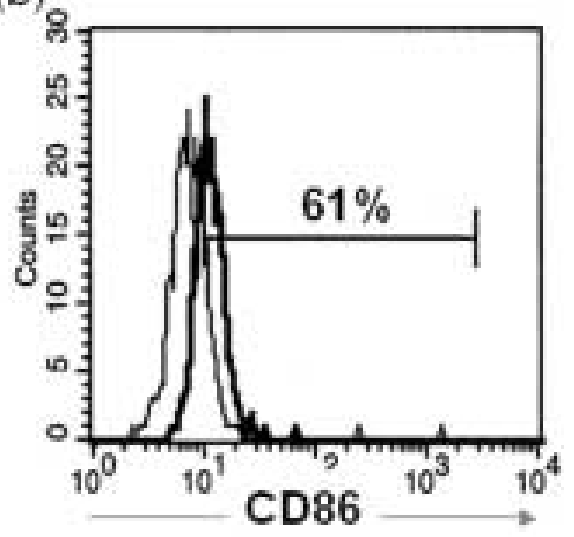

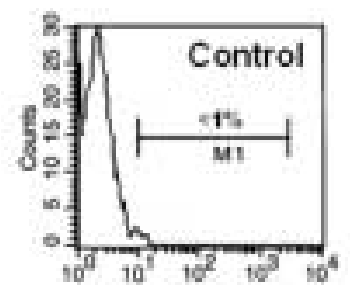

Fig. 3: Expresión de CD80 y CD86 en CEC. Los histogramas muestran la intensidad de fluorescencia para CD80 (a) y CD86 (b) en CEC no infectadas (línea delgada) y en CEC infectadas (línea gruesa) a las 72 h de cultivo. Los histogramas fueron realizados a partir de $R I+R 2$, por lo que los datos presentados corresponden exclusivamente a $C E C$ $C K+$. Los datos presentados aquí provienen de CEC expandidas a partir de 3 botones corneales de individuos no relacionados (Ver sección de material y métodos), y de cada expansión celular se hicieron ensayos por triplicado. 

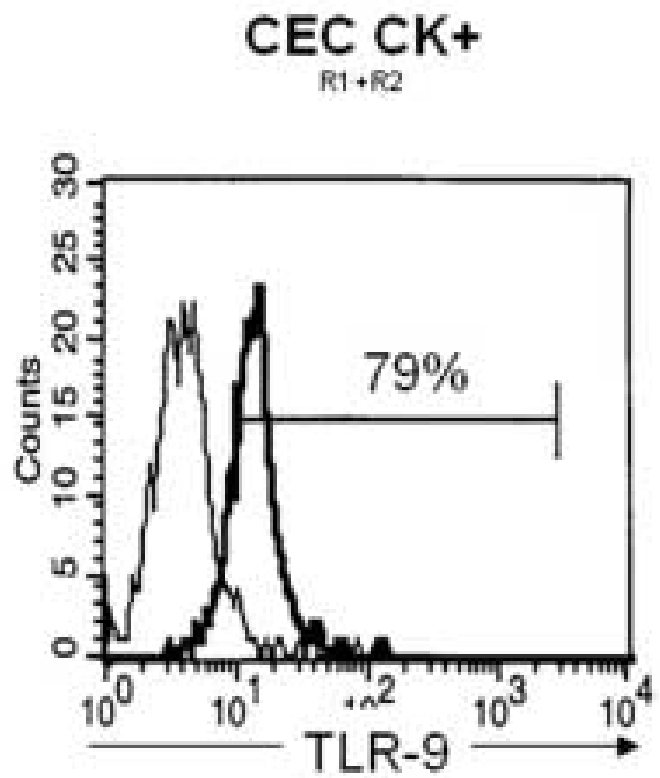

Fig. 4: Expresión de TLR-9 en CEC. El histograma muestra la intensidad de fluorescencia para TLR-9 en CEC no infectadas (línea delgada) y CEC infectadas (línea gruesa) a las $72 \mathrm{~h}$ de cultivo. El histograma fue realizado a partir de $R 1+R 2$, por lo que los datos presentados corresponden exclusivamente a CEC CK+. Los datos presentados aquí provienen de CEC expandidas a partir de 3 botones corneales de individuos no relacionados (Ver sección de material y métodos), y de cada expansión celular se hicieron ensayos por triplicado.

\section{DISCUSIÓN}

En ausencia de infección el ojo utiliza varios mecanismos que mantienen la tolerancia ante $\mathrm{Ag}$ propios y evitan la activación de la respuesta inmunitaria (1-3,8-10). En este contexto, una respuesta inflamatoria que en otro sitio sería protectora, en el ojo generaría más daño que beneficio, de ahí surgió el interés por estudiar algunos de los mecanismos inmunológicos con los que cuentan las CEC cuan-

Tabla II. Cinética de expresión de TLR-9 en CEC CK+ en los diferentes días de cultivo

\begin{tabular}{lrcc}
\hline & \multicolumn{3}{c}{ TLR-9 } \\
& $24 \mathrm{~h}$ & $48 \mathrm{~h}$ & $72 \mathrm{~h}$ \\
\hline CEC Infectadas & $62 \pm 8,1^{*}$ & $63,3 \pm 7,2^{* *}$ & $80 \pm 1,2 \#$ \\
CEC No infectadas & $5 \pm 1,2^{*}$ & $4 \pm 1^{* *}$ & $5 \pm 1 \#$ \\
\hline
\end{tabular}

Resultados expresados en porcentaje \pm error estándar. *: $\mathrm{p}=0,002$; **: $\mathrm{p}=0,001 ; \#: \mathrm{p}<0,001$.

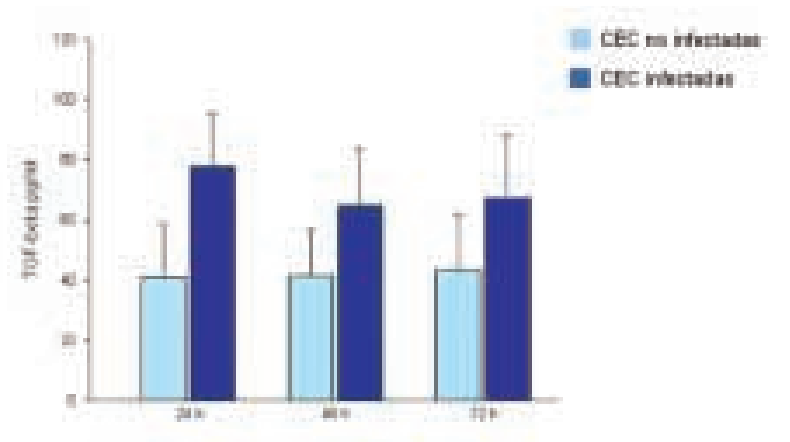

Fig. 5: Concentración de TGF- $\beta$ en sobrenadante de las células en cultivo. Gráfica de barras que corresponde a la concentración de TGF- $\beta$ en los diferentes días de cultivo; CEC no infectadas (azul cielo), CEC infectadas (azul marino). Resultados expresados en $\mathrm{pg} / \mathrm{ml}$.

do son infectadas por virus. Para alcanzar nuestro objetivo se infectaron CEC humanas con Ad5. La infección con Ad5, ha sido utilizada ampliamente como modelo de infección por adenovirus en animales $(21,22)$ pero hay pocos estudios con CEC humanas; Trousdale MD et al. (23) demostraron que las CEC de conejo y humanas pueden ser infectadas por Ad5, alcanzando la máxima cantidad de partículas virales entre las 48 y $72 \mathrm{~h}$. Es por esto que los cultivos celulares en nuestro estudio fueron conducidos hasta las $72 \mathrm{~h}$. Durante el tiempo de cultivo, las CEC fueron perdiendo su morfología epitelial y adquirieron una morfología fibroblástica. Este fenómeno ocurre frecuentemente en cultivos primarios de células corneales y está mediado por factores de crecimiento locales como el de crecimiento fibroblástico (FGF), crecimiento derivado de plaquetas (FGDP) y el TGF- $\beta$ que pueden inducir, dependiendo de su concentración, la expresión de moléculas que permiten diferenciar fenotípicamente a las células en fibroblastos o miofibroblastos (24). En nuestro estudio se determinó la concentración de TGF- $\beta$ que se encontró presente en los cultivos celulares y es probable que esta citocina pudiera estar involucrada con los cambios morfológicos observados, como la adquisición de una forma fibroblástica o con los cambios fenotípicos, como la pérdida de expresión de citoqueratina ya que Ogawa E. et al. (25) reportaron datos similares, utilizando un modelo de infección adenoviral latente en células del epitelio respiratorio. En ese mismo trabajo los autores observaron que justo por debajo de las células infectadas por Ad existe una remode- 


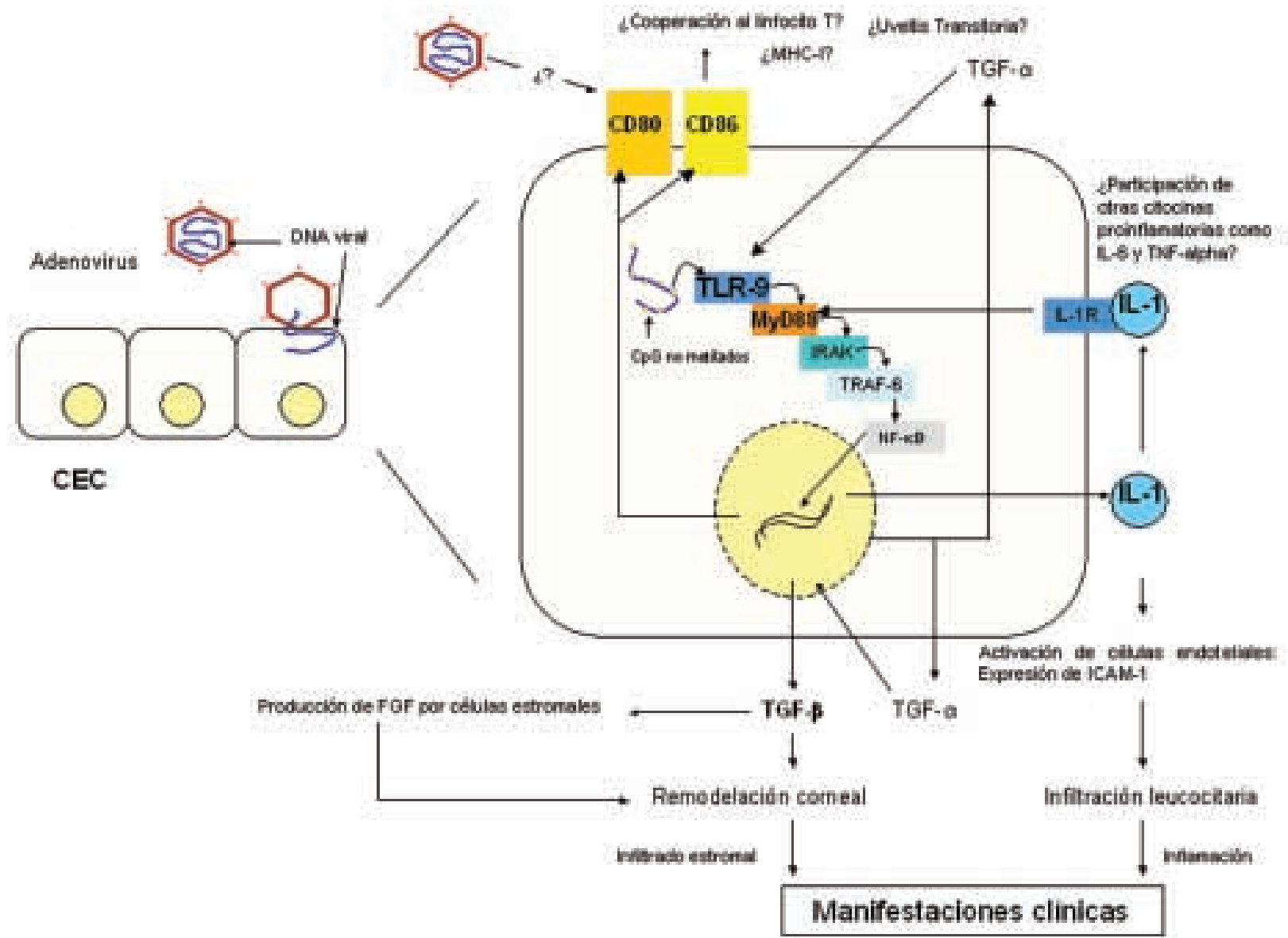

Fig. 6: Secuencia hipotética de eventos moleculares durante una infección por adenovirus.

lación del estroma a nivel subepitelial y ellos sugieren que esta remodelación podría estar mediada por el TGF-beta (24).

La interacción de B7 con su ligando natural (CD28) expresado en linfocitos $\mathrm{T}$, es crítico en el proceso de activación Ag-específica (26) sin embargo es reconocido que las CEC no expresan moléculas coestimulatorias $(1,4)$ por lo que en este trabajo se investigó si las CEC infectadas por Ad5 podían inducir en su superficie moléculas de la familia B7. Nuestros experimentos mostraron que las CEC infectadas in vitro con Ad5 pueden expresar tanto CD80 como CD86. Previamente a este trabajo se había comunicado que las únicas células con capacidad de expresar moléculas coestimulatorias en córnea eran las células dendríticas y las células de Langerhans en respuesta a un estímulo (6-7, 28). Las citocinas proinflamatorias [IL-1, IL-6 y Factor de necrosis tumoral alfa (TNF-a)] están involucra- das con la expresión de moléculas B7 en células dendríticas (29) en células epiteliales durante estímulos crónicos, como la dermatitis atópica $(13,30)$ o durante infecciones virales, en el epitelio respiratorio (14). En este trabajo no se determinaron las concentraciones de estas citocinas en el sobrenadante, sin embargo es probable que estas estuvieran presentes en el microambiente ya que Chang $\mathrm{CH}$ et al han demostrado que una infección por Ad puede inducir la producción de grandes cantidades de IL1 en las CEC infectadas (12).

Por último, en este estudio también se evaluó la expresión de TLR-9 en CEC, los receptores tipo Toll son una familia de receptores involucrados con la respuesta inmune innata y tienen como ligandos estructuras moleculares conservadas como lipopolisacárido, flagelina, entre otros (16). Recientemente se comunicó la expresión de TLR-2, TLR-4 y TLR9 en la córnea de ratón (31). TLR-9 reconoce el 
CpG no metilado presente en el ADN viral (16) y la vía de activación inducida por TLR-9 puede inducir la producción de citocinas proinflamatorias $(32,33)$.

Los resultados de este estudio podrían tener implicaciones clínico-patológicas importantes en la queratoconjuntivitis viral, es conocido que la queratoconjuntivitis por adenovirus es la más frecuente de las infecciones virales de la superficie ocular (11). Se caracteriza por hiperemia conjuntival, sensación de cuerpo extraño, epifora, reacción folicular en la conjuntiva palpebral, edema palpebral y linfadenopatía. En la córnea se observa una queratitis epitelial fina difusa perceptible con fluoresceína, que puede persistir de 2 a 3 semanas. Estas lesiones pueden evolucionar, dependiendo de la actividad viral, a una queratitis epitelial focal, caracterizada por opacidades subepiteliales blancas y focales justo por debajo de las lesiones epiteliales, esta queratitis puede persistir 1 a 2 semanas y en algunos casos puede asociarse con una uveitis anterior transitoria moderada. Aproximadamente 2 semanas después del inicio de los síntomas aparece un infiltrado estromal anterior que puede persistir durante semanas o años (34).

Los mecanismos moleculares involucrados en la generación de los datos clínicos podrían tener la siguiente secuencia hipotética: A. La infección por Ad en CEC interacciona con su ligando intracelular (TLR-9), esta molécula interacciona con los $\mathrm{CpG}$ no metilados del virus e induce la activación celular. La activación vía TLR-9 induce la producción de citocinas proinflamatorias, como IL-1. Esta citocina es el principal mediador de las reacciones inflamatorias de la superficie ocular, ya que regula la activación de células endoteliales en quienes induce la expresión de moléculas de adhesión intercelular-1 (ICAM-1), lo que a su vez favorece el reclutamiento leucocitario al lugar de la infección viral (12); B. La IL-1, junto con IL-6 y TNF- $\alpha$, induce la expresión de moléculas B7 en células dendríticas (29-30), probablemente estas citocinas estén involucradas en la inducción de moléculas B7 en las CEC infectadas por Ad. C. La expresión de CD80 y CD86 en las CEC puede tener dos consecuencias, la primera favorecer la activación de linfocitos $\mathrm{T}$ específicos del virus, lo que necesitaría de la coexpresión de MHC-I, pero este mismo mecanismo puede activar a clonas autoreactivas como ha sido demostrado en queratitis herpéticas (35) generando la uveitis moderada transitoria. La segunda posibilidad es que el virus utilice a las moléculas
B7 como ligandos para infectar a más CEC activadas, lo que se ha demostrado al menos para Ad3 (36). D. El microambiente también juega un papel importante en la respuesta inmune antiviral, el TGF- $\alpha$ producido normalmente por las CEC induce tanto la expresión de TLR-9 y TGF- $\beta$. (37). El TGF-b a su vez, induce la producción de FGF por los fibroblastos estromales (38) ambas citocinas, TGF- $\beta$ y FGF, podrían favorecer la remodelación (25) a nivel corneal lo que correspondería con las lesiones subepiteliales (26), y con las opacidades del estroma corneal (fig. 6).

En resumen, los resultados del presente estudio sugieren que las CEC pueden expresar CD80, CD86 y TLR-9 durante una infección viral, el conocimiento de las implicaciones clínico-patológicas sugeridas aquí, puede ser utilizado como blanco para futuras investigaciones o intervenciones terapéuticas en las queratitis virales.

\section{AGRADECIMIENTOS}

Se agradece a la Sra. Verónica Romero Martínez y a Pablo Ottalengo por el apoyo técnico recibido.

\section{BIBLIOGRAFÍA}

1. Streilein JW. Ocular immune privilege: therapeutic opportunities from an experiment of nature. Nat Rev Immunol 2003; 3: 879-889.

2. Cursiefen C, Chen L, Dana MR, Streilein JW. Corneal lymphangiogenesis: evidence, mechanisms, and implications for corneal transplant immunology. Cornea 2003; 22: 273-281.

3. Stuart PM, Griffith TS, Usui N, Pepose J, Yu X, Ferguson TA. CD95 ligand (Fas-L)-induced apoptosis is necessary for corneal allograft survival. J Clin Invest 1997; 99: 396402.

4. Streilein JW. New thoughts on the immunology of corneal transplantation. Eye 2003; 17: 943-948.

5. Prilliman KR, Lemmens EE, Palioungas $G$, Wolfe TG, Allison JP, Sharpe AH, et al. Cutting edge: a crucial role for B7-CD28 in transmitting $T$ help from APC to CTL. $J$ Immunol 2002; 169: 4094-4097.

6. Hamrah $P$, Liu $Y$, Zhang $Q$, Dana MR. The corneal stroma is endowed with a significant number of resident dendritic cells. Invest Ophthalmol Vis Sci 2003; 44: 581-589.

7. Novak N, Siepmann K, Zierhut M, Bieber T. The good, the bad and the ugly--APCs of the eye. Trends Immunol 2003; 24: 570-574.

8. Stein-Streilein J, Streilein JW. Anterior chamber associated immune deviation (ACAID): regulation, biological relevance, and implications for therapy. Int Rev Immunol 2002; 21: 123-152. 
9. Wilbanks GA, Streilein JW. Characterization of suppresor cells in anterior chamber-associated immune deviation (ACAID) induced by soluble antigen. Evidence of two functionally and phenotypically distinct T-suppressor cell populations. Immunology 1990; 71: 383-389.

10. Kosiewicz, MM, Alard P, Streilein JW. Alterations in cytokine production following intraocular injection of soluble protein antigen: impairment in IFN-gamma and induction of TGF-beta and IL-4 production. J Immunol 1998; 161: 5382-5390.

11. Cheung D, Bremner J, Chan JT. Epidemic kerato-conjunctivitis--do outbreaks have to be epidemic? Eye 2003; 17: 356-363.

12. Chang $C H$, Huang $Y$, Issekutz, AC, Griffith $M$, Lin $K H$, Anderson $R$. Interleukin-1alpha released from epithelial cells after adenovirus type 37 infection activates intercellular adhesion molecule 1 expression on human vascular endotheial cells. J Virol 2002; 76: 427-431.

13. Esche $C$, de Benedetto A, Beck LA. Keratinocytes in atopic dermatitis: inflammatory signals. Curr Allergy Asthma Rep 2004; 4: 276-284.

14. Kim J, Myers AC, Chen L, Pardoll DM, Truong-Tran QA, Lane AP, et al. Constitutive and inducible expression of $b 7$ family of ligands by human airway epithelial cells. Am J Respir Cell Mol Biol 2005; 33: 280-289.

15. Hu M, Dutt J, Arrunategui-Correa V, Baltatzis S, Foster $C S$. Cytokine mRNA in BALB/c mouse corneas infected with herpes simplex virus. Eye 1999; 13: 309-313.

16. Janssens $S$, Beyaert $R$. Role of Toll-like receptors in pathogen recognition. Clin Microbiol Rev 2003; 16: 637-646.

17. Ban Y, Cooper LJ, Fullwood NJ, Nakamura T, Tsuzuki M, Koizumi N, et al. Comparison of ultrastructure, tight junction-related protein expression and barrier function of human corneal epithelial cells cultivated on amniotic membrane with and without air-lifting. Exp Eye Res 2003; 76: 735-743.

18. Johansson ME, Andersson MA, Thorner PA. Adenoviruses isolated in the Stockholm area during 1987-1992: restriction endonuclease analysis and molecular eidemiology. Arch Virol 1994; 137: 101-115.

19. Lee MS, Cohen B, Hand J, Nokes DJ. A simplified and standardized neutralization enzyme immunoassay for the quantification of measles neutralizing antibody. J Virol Methods 1999; 78: 209-217.

20. Kivela T, Uusitalo M. Structure, development and function of cytoskeletal elements in non-neuronal cells of the human eye. Prog Retin Eye Res 1998; 17: 385-428.

21. Gordon YJ, Romanowski E, Araullo-Cruz T. An ocular model of adenovirus type 5 infection in the NZ rabbit. Invest Ophthalmol Vis Sci 1992; 33: 574-580.

22. Trousdale MD, Nobrega R, Stevenson D, Nakamura T, dos Santos PM, Labree L, et al. Role of adenovirus type 5 early region 3 in the pathogenesis of ocular disease and cell culture infection. Cornea 1995; 14: 280-289.

23. Wood RL, Trousdale MD, Stevenson D, Azzarolo AM, Mircheff AK. Adenovirus infection of the cornea causes histopathologic changes in the lacrimal gland. Curr Eye Res 1997; 16: 459-466.

24. Trousdale MD, Nobrega R, Wood RL, Stevenson D, dos Santos PM, Klein D, et al. Studies of adenovirus-induced eye disease in the rabbit model. Invest Ophthalmol Vis Sci 1995; 36: 2740-2748.

25. Jester JV, Ho-Chang J. Modulation of cultured corneal keratocyte phenotype by growth factors/cytokines control in vitro contractility and extracellular matrix contraction. Exp Eye Res 2003; 77: 581-592.

26. Ogawa E, Elliott WM, Hughes F, Eichholtz TJ, Hogg JC, Hayashi S. Latent adenoviral infection induces production of growth factors relevant to airway remodeling in COPD. Am J Physiol Lung Cell Mol Physiol 2004; 286: L189-L197.

27. Ward SG. CD28: a signalling perspective. Biochem J 1996; 318:361-377.

28. Hamrah P, Huq SO, Liu Y, Zhang Q, Dana MR. Corneal immunity is mediated by heterogeneous population of antigen-presenting cells. J Leukoc Biol 2003; 74: 172-178.

29. Yamada N, Katz SI. Generation of mature dendritic cells from a CD14+ cell line (XS52) by IL-4, TNF-alpha, IL-1 beta, and agonistic anti-CD40 monoclonal antibody. J Immunol 1999; 163: 5331-5337.

30. Burns R, Luzina I, Nasir A, Haidaris CG, Barth RK, Gaspari AA. Keratinocyte-derived, CD80-mediated costimulation is associated with hapten-specific IgE production during contact hypersensitivity to TH1 haptens. J Allergy Clin Immunol 2005; 115: 383-390.

31. Johnson AC, Heinzel FP, Diaconu E, Sun Y, Hise AG, Golenbock D, et al. Activation of toll-like receptor (TLR)2, TLR4, and TLR9 in the mammalian cornea induces MyD88-dependent corneal inflammation. Invest Ophthalmol Vis Sci 2005; 46: 589-595.

32. Akira $S$, Takeda $K$. Toll-like receptor signaling. Nat Rev Immunol 2004: 4: 499-511.

33. Liu L, Zhou X, Shi J, Xie X, Yuan Z. Toll-like receptor-9 induced by physical trauma mediates release of cytokines following exposure to $\mathrm{Cp} G$ motif in mouse skin. Immunology 2003; 110: 341-347.

34. Kanski JJ. Oftalmología clínica. V edición. Madrid: Elsevier España, S.A; 2004; 68-69.

35. Gangappa S, Deshpande SP, Rouse BT. Bystander activation of CD4+ T cells accounts for herpetic ocular lesions. Invest Ophthalmol Vis Sci 2000; 41: 453-459.

36. Short JJ, Pereboev AV, Kawakami Y, Vasu C, Holterman MJ, Curiel DT. Adenovirus serotype 3 utilizes CD80 (B7.1) and CD86 (B7.2) as cellular attachment receptors. Virology 2004; 322: 349-359.

37. Miller LS, Sorensen OE, Liu PT, Jalian HR, Eshtiaghpour $D$, Behmanesh BE, et al. TGF-alpha regulates TLR expression and function on epidermal keratinocytes. $J$ Immunol 2005; 174: 6137-6143.

38. Kay EP, Lee MS, Seong GJ, Lee YG. TGF-beta s stimulate cell proliferation via an autocrine production of $F G F-2$ in corneal stromal fibroblasts. Curr Eye Res 1998; 17:286-293. 